\title{
The Boundaries of Sequential Modulations: Evidence for Set-Level Control
}

\author{
Eliot Hazeltine \\ University of Iowa
}

\author{
Erin Lightman, Hillary Schwarb, and \\ Eric H. Schumacher \\ Georgia Institute of Technology
}

\begin{abstract}
We examined the sequential modulation of congruency effects using a task in which the irrelevant information shares the same stimulus dimensions as the relevant information but is presented at an earlier time. In Experiment 1, sequential modulations were observed within a stimulus modality but not between stimulus modalities. In Experiment 2, sequential modulations were observed across two sets of visual stimuli, even though the two sets involved distinct stimulus dimensions. Experiment 3 used the same stimuli as Experiment 2, but required different responses for the two sets of stimuli. In this case, sequential modulations were specific to the stimulus set. In Experiment 4, two stimulus sets were presented along two stimulus modalities, and sequential modulations crossed both set and modality boundaries. These results suggest that control processes obey flexible boundaries defined by task constraints.
\end{abstract}

Keywords: conflict adaptation, cognitive control, response competition, task representation

Selecting task-relevant information and making the appropriate response is a fundamental aspect of goal-directed behavior. To study the processes supporting this ability, psychologists often turn to response conflict tasks in which the stimulus display offers multiple sources of information, some consistent with the appropriate response and some not. For example, the Stroop, Simon, and flanker tasks have all been the focus of considerable research for many years, leading to rigorous models of how the human information processing system resolves conflict between competing responses in these situations.

In each of these tasks, response conflict is measured by increased reaction time (RT) on trials in which task-irrelevant information indicates an incorrect response (incongruent trials) compared to trials in which the task-irrelevant information indicates the correct response (congruent trials). Recently, researchers have begun to focus on sequential modulations of the congruency effect that are observed in all three of these tasks. First reported by Gratton and colleagues (1992, see also Stürmer, Leuthold, Soetens, Schröter, \& Sommer, 2002) in the flanker task, smaller (or even reversed) differences between incongruent and congruent trials are observed following incongruent trials than following congruent trials. That is, the congruency effect is reduced after an incongruent trial compared to after a congruent trial. In the present paper, we refer to these changes in congruency effects as sequential modulations (as in Kunde, 2003; Wendt, Kluew, \& Peters, 2006).

An appealingly straightforward account of sequential modulations holds that they reflect changes in how selective attention is

This article was published Online First July 18, 2011.

Eliot Hazeltine, Department of Psychology, University of Iowa; Erin Lightman, Hillary Schwarb, and Eric H. Schumacher, School of Psychology, Georgia Institute of Technology.

The authors are grateful to Mike Wendt and Peter Wühr for their extremely helpful comments on the manuscript.

Correspondence concerning this article should be addressed to Eliot Hazeltine, Department of Psychology, University of Iowa, 11 Seashore Hall E, Iowa City, IA 52242-1407. E-mail: eliot-hazeltine@uiowa.edu deployed given the detection of conflict, a hypothesis sometimes termed conflict adaptation. In their groundbreaking paper, Botvinick, Braver, Barch, Carter, \& Cohen (2001) proposed a four-part model of control in which conflict is resolved by manipulating input attention to different dimensions or features within the stimulus display. According to this conflict monitoring hypothesis, a conflict monitoring process detects increases in competition among response alternatives and signals the attention system to increase the weightings of relevant sources of information and/or attenuate irrelevant sources. Thus, on incongruent trials, the attention system increases the difference between the attentional weightings of relevant and irrelevant signals more than it does on congruent trials. This state persists to the subsequent trial, where the irrelevant stimuli therefore have a reduced effect. This elegant framework allows the model to capture sequential modulations across a range of tasks. Moreover, it can account for them without invoking an intelligent agent that makes strategic decisions or keeps track of the different types of trials. That is, it avoids the so-called "homunculus problem" (Logan, 2003; Monsell \& Driver, 2000). The model simply detects conflict and adjusts attention, so that the task-relevant source of information is appropriately weighted on the subsequent trial. This is accomplished without having to sort out the various sources of activation for the candidate responses (but see Akçay \& Hazeltine, 2011).

A critical issue in determining the source of sequential modulations is the role that individual stimulus features play. It has been pointed out that in typical two-choice tasks, congruent trials that follow congruent trials, and incongruent trials that follow incongruent trials, involve either exact repetitions of stimulus features or complete pairings of features that were not present on the previous trial (Hommel, Proctor, \& Vu, 2004; Mayr, Awh, \& Laurey, 2003). In contrast, congruent trials that follow incongruent trials, and incongruent trials that follow congruent trials involve a repeated feature paired with a novel feature. This latter situation may induce a binding cost that increases RTs, leading to a sequential modulation effect without requiring any type of control mechanism. That is, when each stimulus dimension has just two possible values 
and the congruency switches on consecutive trials, then one feature from the previous trial must repeat and the other must change. This partial repetition may require participants to do extra work to bind the various features of the stimulus into a unitary percept (see Hommel, 1998). To eliminate this alternative explanation, researchers have employed tasks with more than two stimuli and two responses, so that all combinations of congruent and incongruent trials can be composed of novel features. In general, robust sequential modulations are still observed under these conditions (e.g., Akçay \& Hazeltine, 2007, 2008, 2011; Funes, Lupiáñez, \& Humphreys, 2010; Ullsperger, Bylsma, \& Botvinick, 2005), suggesting that they arise, at least in part, independently from the effects of feature repetitions and feature binding.

\section{Sequential Modulations and the Architecture of Cognitive Control}

The present experiments aim to probe the structure of executive control processes by examining sequential modulations in a priming task. Our goal is to identify the factors that cause the congruency of one trial to affect the magnitude of the congruency effect of a subsequent trial. By doing so, we can trace the changes undergone by response selection processes when they are confronted by either a congruent or incongruent trial.

The priming task we use is a variant of the flanker task in which the distractors differ from the targets temporally rather than spatially (cf., Kiesel, Kunde, \& Hoffman, 2006; Kunde, 2003; Kunde \& Wühr, 2006). In this temporal flanker task, participants are instructed to ignore the identity of the first centrally presented stimulus (i.e., the flanker) and respond only to the second centrally presented stimulus (i.e., the target). We use the term "temporal flanker" to emphasize that primes and targets are physically identical and it is only the order of their presentation that differentiates one from the other. As in the standard flanker task, the flanker stimulus can be congruent and indicate the same response as the target, or it can be incongruent and indicate a different response.

Because the target and flanker are presented at distinct points in time, it is possible to use both visual and auditory stimuli with the temporal flanker task without relying on the participants' ability to spatially localize the stimuli to differentiate the target and flanker. Moreover, the same task instructions can be used for both types of stimuli: "respond to the second item and ignore the first." Furthermore, because the visual and auditory stimuli can be conceptually related, the same S-R mappings can be used across the two stimulus sets as well. In this way, the critical features of the tasks (viz., S-R dimensions) are identical for tasks using auditory and visual stimuli.

With this task, we address two fundamental questions relating to the source of sequential modulations. First, we examine whether sequential modulations are observed when the irrelevant and relevant stimuli have the same physical properties but are presented in sequence rather than simultaneously. Second, we determine the locus of the changes in task processing by using four choice tasks in which the effects of modulating the weighting of specific features can be dissociated from the effects of modulating the weighting of a stimulus dimension. We describe each of these issues below.

\section{Sequential Modulations and Attention}

In their influential model, Botvinick et al. (2001) proposed that sequential modulations reflect changes in the weighting of various sources of information for response selection. Egner, Delano, and Hirsch (2007) extended this idea by testing the boundaries of sequential modulations and concluded that they reflected changes in the weightings of particular stimulus dimensions (e.g., color or location). This proposal accounts for many aspects of sequential modulations (see below), but it also suggests that they should not be observed when the relevant and irrelevant stimuli involve the same stimulus dimensions, as in previous priming studies (e.g., Kunde, 2003; Kunde \& Wühr, 2006). That is, when the target is presented in the same location and immediately after the distractor, the physical properties, apart from the relative timing of the onsets and offsets, cannot be selected to differentiate the target and distractor. Under these conditions, to attend more to the target than to the distractor requires weighting the input relatively more for the second stimulus than for the first. However, "first-ness" and "second-ness" are generally not considered preattentive features like "red" or "vertical" and relative order is generally not considered a stimulus dimension like color and orientation. Rather, relative order depends on task context and is not an inherent property of the stimulus. Thus, while the brain clearly represents relative order to successively perform these sorts of tasks, it is unlikely that the sort of feature maps used to code color and orientation are employed.

If sequential modulations result simply from carry-over of the attentional state from one trial to the next, then experiments in which the irrelevant information precedes the relevant information but is presented along the same stimulus dimensions should produce a reversed sequential modulation. This prediction holds because the first stimulus on the next trial is always irrelevant (i.e., the flanker) and greater attention to it should increase the congruency effect. On incongruent trials, the first stimulus provides conflicting information that must be suppressed relative to the second stimulus. To overcome this, one might assume that participants allocate more attention to the second stimulus than the first, leaving the attentional weights for the location and physical properties of the stimulus relatively high when the next trial begins. In contrast, on congruent trials, both the first and second stimulus indicate the correct response, so there is no need to weight the latter part of the sequence more heavily than the first. Therefore, if the previous trial is incongruent, then attention to the stimulus location should be weighted relatively greater, so as to overcome the incorrect response activation associated with the flanker. This state, according to the attentional carryover account, should persist until the beginning of the subsequent trial, when the flanker appears first, thereby causing a greater congruency effect. Conversely, if the previous trial is congruent, then attention to the stimulus location when the target is presented will be weighted relatively less, because the flanker already has indicated the correct response. Therefore, trials following congruent trials should have less attention allocated to the stimulus location at the beginning of the trial (i.e., when the flanker appears) and therefore should show less congruency effect. In this way, the attentional account predicts reversed sequential modulations for the priming temporal flanker tasks. 
Two priming studies have reported sequential modulations using primes that differed in terms of size (Kunde, 2003; Kunde \& Wühr, 2006), which is considered a stimulus dimension and thus poses no problem for an attentional carryover account of sequential modulations. A third study (Kiesel et al., 2006) did not evaluate sequential modulations based the relationship between the prime and the target. Rather, Kiesel and colleagues (2006) examined sequential modulations of a congruency effect when the target stimulus, a number, was associated with both a magnitude task and a parity task. Congruency was determined by the relationship of the responses to the target for the two possible tasks (i.e., was the response the same for the parity and magnitude tasks). Intriguingly, a significant sequential modulation was observed. Because the source of the irrelevant information is the same as the source of the relevant information in this case, changes in input attention cannot account for the pattern of results. In the present experiments, we examine whether adjustments in post-perceptual processes can account for sequential modulations when conflicting responses are indicated by distinct stimuli.

With the temporal flanker task, we will test whether sequential modulations are observed when the relevant and irrelevant information occur along the same stimulus dimensions and the irrelevant information is easily detected by the participant. It is possible that the attentional settings are implemented in a sophisticated manner, such as in reference to the relative order of the stimuli rather than simply in terms of their locations in space or some other perceptual dimension. However, this sophistication requires attention to be controlled in relation to task-defined parameters rather than simply in relation to the perceptual dimensions, moving away from the appeal of many of the elegant models of sequential modulations (e.g., Botvinick et al., 2001; Blais, Robidoux, Risko, \& Besner, 2007; Notebaert \& Verguts, 2008). If attentional settings are defined relative to task-defined events, then task representations must play a major role in sequential modulations. In other words, if the traditional form of sequential modulations are observed in the temporal flanker task, they must result from changes in the processing of specific items (e.g., the letter ' $A$ ') or sets (e.g., the first stimulus of the presented pair), rather than features or dimensions.

\section{The Locus of Sequential Modulations}

A second issue relating to sequential modulations is whether they reflect local or global control processes (Akçay \& Hazeltine, 2007, 2008; Egner, Delano \& Hirsch, 2007; Freitas, Bahar, Yang, \& Banai, 2007; Funes et al., 2010; Kunde \& Wühr, 2006; Notebaert \& Verguts, 2008; Wendt et al., 2006). Local control refers to sequential modulations that are specific to a task or stimulus dimension, whereas global control refers to changes in processes that affect subsequent processing regardless of whether the task and irrelevant stimulus dimension are the same. While the preponderance of the available evidence suggests that the control is implemented locally, the exact level remains largely undetermined. For example, sequential modulations of congruency effects do not cross from a flanker task to a Simon task (Funes et al., 2010), or even from one Simon task to a second, distinct Simon task (Akçay \& Hazeltine, 2008), so it appears that the conflictinduced changes in processing are specific to some components of the particular task. However, it is unclear whether the changes relate to individual features (e.g., Blais et al., 2007), stimulus dimensions (Egner, Delano, \& Hirsch, 2007), or some high-level aspect of the task representation (e.g., Akçay \& Hazeltine, 2008). In the present study, we differentiate among three potential levels of control: item-level control, set-level control, and global control.

Item-level control refers to changes in processing associated with a particular stimulus; that is, control processes adjust the weightings of individual items or features. Blais and colleagues (Blais, Robidoux, Risko, \& Besner, 2007) propose a modification of Botvinick and colleagues' (2001) conflict monitoring model such that control is implemented at the level of specific features rather than at the level of the dimensions at which those features occur. In other words, rather than assuming that the weights associated with a type of information (e.g., color or location) are changed in response to the detection of conflict, Blais and colleagues assume that the weights associated with individual features (e.g., red or left) are changed.

This modified (feature-based) version of the model is better able to account for the item-specific proportion-congruent effect (Jacoby, Lindsay \& Hessels, 2003) than the original dimensionbased version. Moreover, localizing changes to the associations of individual features may also explain why sequential modulations are restricted to task-sets (e.g., Akçay \& Hazeltine, 2008) without having to assume that control processes act in a task-specific manner. That is, feature-based control accounts can explain findings of local control without resorting to multiple control processes, because they assume that the weight associated with a particular feature is adjusted in response to conflict and these adjustments would not affect performance of other task-sets involving distinct sets of features (but see Akçay \& Hazeltine, 2011).

Set-level control refers to changes in processing associated with a stimulus set. For example, if the location of a stimulus indicates the incorrect response on a trial, then the location of the stimulus on the next trial will have less influence on response selection than following a trial in which the location indicated the correct response. This effect does not depend on whether the locations on the current and previous trials match, because the changes are associated with all possible locations. However, modulations of the weighting of location do not affect the weightings of other stimulus dimensions (see Akçay \& Hazeltine, 2011; Egner, 2008; Funes et al., 2010). In the existing literature, sets are usually treated as stimulus dimensions (e.g., Botvinick et al., 2001; Egner \& Hirsch, 2005; Funes et al., 2010; Notebaert \& Verguts, 2008), but, as discussed previously, this formulation will not work for the temporal flanker task because the relevant and irrelevant information occurs along the same dimensions. Nonetheless, the flankers and targets are easily identifiable on each trial and it is possible that the congruency of one flanker affects the processing of subsequent flankers belonging to the same set.

Finally, global control refers to changes in processing that generalize across tasks and sets. Thus, the congruency of a trial affects the processing of irrelevant information on the subsequent trial regardless of the source of the irrelevant information. Support for this account was provided by Freitas et al. (2007). These researchers found that sequential modulations were observed between the flanker and color Stroop tasks and between the flanker and spatial Stroop tasks, even though the two tasks used very different stimulus sets (see also, Fernandez-Duque \& Knight, 2008). 
To differentiate these various forms of control, we use two four-choice tasks in four experiments. When there are only two possible items, the suppression of a particular item and the suppression of the entire set will produce similar patterns of results, given that every trial will either repeat the item of the previous trial or use the previously suppressed item (see, Hommel, Proctor, \& Vu, 2004; Mayr, Awh, Laurey, 2003). Under such conditions, it is difficult to distinguish between item-level control (or item-level binding costs) and set-level control. However, when there are four possible items, performance on trials with the previously suppressed items can be compared to performance on trials with novel items that were not present on the previous trial, allowing for the dissociation of item-level and set-level effects.

To distinguish between set-level and global control, we use two tasks that, in Experiment 1, involve distinct stimulus modalities. If sequential modulations are observed across stimulus modalities, this will suggest that control processes act in a global fashion, affecting performance across a range of input systems. In contrast, if sequential modulations are only observed when the modality repeats, this will indicate some form of set-level control. This might entail control processes that are specific to a stimulus modality or control processes whose boundaries are determined by the stimulus-response mappings or other task properties.

To preview our findings, Experiment 1 provides evidence for local control-that is, the congruency of trials with visual stimuli does not appear to affect the magnitude of the congruency effect for trials with auditory stimuli and vice versa. The subsequent experiments aim to identify the nature of the boundaries for sequential modulations, whether they relate to perceptual processes and whether they are sensitive to post-perceptual factors. In this way, we can discriminate among accounts that rely on changes in the processing of individual stimulus features, accounts that rely on changes specific to stimulus modalities or dimensions, and accounts that are based on changes in higher-level task representations.

\section{Experiment 1}

In Experiment 1, we tested whether sequential modulations would be observed in the temporal flanker task, in which targets and distractors occurred along the same stimulus dimensions. Furthermore, we used two distinct modalities, vision and audition, each with four possible targets. In this way, we could independently assess global sequential modulations (i.e., reductions in congruency effects across modality), set-level sequential modulations (i.e., reductions in congruency effects within a task but involving stimuli that had not appeared on the previous trial), and item-level sequential modulations (i.e., reductions in congruency effects depending on the congruency of the trial the last time the same flanker was used).

Both the visual and auditory stimuli were the letters 'A,' 'B,' ' $\mathrm{C}$,' and ' $\mathrm{D}$,' and the participants' task was always the same: press the leftmost key for the letter 'A,' the key the second from the left for the letter 'B,' the key the second from the right for the letter ' $\mathrm{C}$ ' and rightmost key for the letter 'D.' In this way, participants did not have to remember distinct S-R mappings, and responding to the visual and auditory stimuli was of similar difficulty.

The critical analyses identified the locus of sequential modulations by examining the interaction between the previous trial's congruency on the current trial's congruency based on two factors. First, we examined set-level control by determining if sequential modulations depend on whether the previous trial uses the same modality as the current trial. Because set is confounded with stimulus modality in this experiment, set-level accounts predict that the magnitude of the congruency effect should be determined by the last trial of the same modality, regardless of the particular stimuli. In contrast, global accounts predict that the magnitude should depend on the last trial, regardless of the particular modality.

To ensure that this analysis identified set-level sequential modulations, and because these types of transitions are not possible on switch trials but may affect performance on repeat trials, we eliminated from the analyses trials in which targets repeated, or flankers repeated, or flankers on the previous trial appeared as targets on the current trial, or targets on the previous trial appeared as flankers on the current trial. Moreover, we counted conceptually related stimuli (e.g., visual "A" and auditory "A") as repetitions, so that the same criteria were applied to switched and repeated modality trials and response repetitions would be eliminated for switches as well as repeated modality trials. These stringent criteria meant that we eliminated approximately half of the data; therefore, we ran the participants in two 1-hr sessions. To maximize the number of trials in each cell of the design and minimize the surprise associated with task switches, we switched tasks predictably every other trial, as in Rogers and Monsell (1995).

Second, we tested for item-based sequential modulations by examining how the history of the flanker affects performance. Item-based accounts predict that the magnitude of the congruency effect should be determined by whether the irrelevant stimulus was congruent or incongruent the last time it appeared in the stimulus display. If control is implemented at the level of the individual item, as in Blais et al. (2007), then the congruency effect should be larger when the flanker was previously incongruent than when it was previously congruent.

Note that the three types of control are not mutually exclusive. It is possible that to uncover evidence for each, given that our tests are independent.

\section{Method}

Participants. Eighteen undergraduate students from Georgia Institute of Technology with ages ranging from 17-24 completed the study to fulfill a requirement for a psychology course. Ten of the participants were female. Two participants were eliminated from the analyses for making errors on more than $10 \%$ of trials.

Apparatus and stimuli. The experiment was conducted on PC compatible computers using E-prime 1.1 software. Participants sat a comfortable distance from the computer monitor, resting their right hands, which were used to make the responses, in front of the computer keyboard. Responses were made using the 'v,' 'b,' 'n,' and ' $\mathrm{m}$ ' keys.

A white fixation cross, $1.5 \mathrm{~cm} \times 1.5 \mathrm{~cm}$, remained present in the center of the screen until $500 \mathrm{~ms}$ before the stimuli. The visual stimuli were white letters, $2 \mathrm{~cm} \times 2 \mathrm{~cm}$, on a black background in the center of the screen where the fixation cross had appeared. The auditory stimuli were .wav files of a male voice speaking the letters 'A,' 'B,' 'C,' and 'D.' Each sound file was edited to last 250 $\mathrm{ms}$ and was presented at a comfortable volume. The flanker 
stimulus was presented first, for $250 \mathrm{~ms}$, followed by a $100 \mathrm{~ms}$ interstimulus interval, which, in turn was followed by the target stimulus, which also lasted $250 \mathrm{~ms}$. The screen remained blank until the participant responded, at which point the fixation cross reappeared for $1000 \mathrm{~ms}$ and the next trial began. The mapping of stimuli to responses was spatially compatible: participants pressed the ' $v$ ' key with their right index fingers when they heard or saw ' $A$,' the ' $b$ ' key with the right middle fingers when they heard or saw 'B,' the ' $n$ ' key with their right ring fingers when they heard or saw ' $\mathrm{C}$ ', and the ' $\mathrm{m}$ ' key with their right pinky fingers when they heard or saw 'D.'

Participants completed two identical sessions of the experiment, usually within a span of three days, each lasting $1 \mathrm{hr}$. Each session consisted of two practice blocks, each consisting of only visual or only auditory stimuli and 10 experimental blocks in which the modality switched every other trial (e.g., visual, visual, auditory, auditory, etc.). Each block consisted of 72 trials. The target and flanker were chosen randomly, with the constraint that half of the auditory and half of the visual trials be congruent.

\section{Results and Discussion}

Reaction time. Trials with incorrect responses (3\%), following incorrect responses, less than $200 \mathrm{~ms}(<1 \%)$, or greater than $2500 \mathrm{~ms}(1 \%)$ were removed from the RT analysis.

The first four blocks of each session (two single modality practice blocks + the first two experimental blocks) were considered practice and not analyzed. To examine sequential modulations in the experimental blocks independently from stimulus repetition effects, all trials in which the target repeated (25\%), the flanker repeated $(25 \%)$, the previous target became the flanker $(26 \%)$, or the previous flanker became the target $(26 \%)$ were eliminated. ${ }^{1}$ Conceptually related stimuli (e.g., an auditory "B" and a visual "B") counted as repeats. We also eliminated trials in which any stimulus appeared for the first time, leaving $48 \%$ of the trials.

Set-level sequential modulations. To determine whether control operated at the set-level, we submitted the data, trimmed of all repetitions, to a four-way ANOVA with modality (visual or auditory), modality switch (same or different modality as previous trial), previous congruency, and current congruency as factors. There were significant main effects of modality switch, $F(1,15)=$ 5.90, $p<.05, M S E=1271.01$, previous congruency, $F(1,15)=$ $4.79, p<.05, M S E=809.26$, and current congruency, $F(1,14)=$ $179.12, p<.0001, M S E=8740.78$. The main effect of switch indicated that participants produced their responses $11 \mathrm{~ms}$ faster when the modality of the current stimulus matched the modality of the previous stimulus $(556 \mathrm{~ms})$ than when it switched $(567 \mathrm{~ms})$. The lack of larger cost here is not surprising given that the stimuli were univalent and the initial irrelevant stimulus may have cued the participant to the relevant task. The main effect of previous congruency indicated that subjects were $8 \mathrm{~ms}$ faster when the previous trial was congruent $(558 \mathrm{~ms})$ than when the previous trial was incongruent $(566 \mathrm{~ms})$. The main effect of congruency indicated that participants responded $152 \mathrm{~ms}$ faster when the flanker stimulus matched the target $(484 \mathrm{~ms})$ than when it did not $(640$ $\mathrm{ms})$. In sum, the temporal flanker task produces a robust congruency effect.

The focus of the present study is sequential modulations of the congruency effect and, critically, there was a sequential modula- tion: Congruency interacted with previous trial congruency, $F(1$, $15)=10.10, p<.01, M S E=1663.87$, indicating that the congruency effect was $33 \mathrm{~ms}$ larger following congruent trials (472 vs. $644 \mathrm{~ms}$ ) than following incongruent trials (496 vs. 636 $\mathrm{ms})$. Thus, the temporal flanker task produces robust and typical sequential modulation, even though the irrelevant and relevant information exist along the same stimulus dimensions. Moreover, because we eliminated all forms of repetitions from one trial to the next, the sequential modulations cannot be attributed to items changing their role (e.g., a flanker becoming a target or vice versa). Finally, because the flanker history (i.e., whether the flanker on the current trial was a target or a flanker in an incongruent trial the last time it appeared) was uncorrelated with the congruency of the previous trial, the sequential modulation cannot be attributed to item-based control.

Critically, there was also a significant interaction between modality switch, previous congruency, and current congruency, $F(1$, $15)=8.90, p<.01, M S E=877.51$. The pattern, depicted in Figure 1, indicates that when the stimulus modality remained the same on consecutive trials, the classic sequential modulation was observed: congruency produced a $193 \mathrm{~ms}$ advantage on trials following congruent trials but only a $139 \mathrm{~ms}$ advantage on trials following incongruent trials, $t(15)=3.68, p<.005$. In contrast, when the stimulus modality switched on consecutive trials, the congruency of the previous trial did not moderate the magnitude of the congruency effect on the present trial (following congruent trials: $141 \mathrm{~ms}$, following incongruent trials: $152 \mathrm{~ms}, t=1.07$, $p>.3)$.

That the typical sequential modulation was observed when the modality repeated indicates that its source is more complex than is suggested by the residual attention explanation. In the temporal flanker task, the relevant and irrelevant information are presented within the same stimulus dimensions and differ only in terms of their relative timing. Therefore, changes in the sensitivity to irrelevant information cannot be explained in terms of attention selecting one dimension over another. That is, because the flanker of the current trial appears in the exact location and along the same stimulus dimensions as the target of the previous trial, enhancing attention to the target to resolve conflict would result in a reversed sequential modulation, as described in the Introduction. Thus, the present findings indicate that sequential modulations stem from changes in task processing that occur at a level that involves the task representation and not simply at the level of attentional selection (cf., Akçay \& Hazeltine, 2008).

That no sequential modulation was observed in the switch modality trials indicates that control appears to be implemented on a local level, consistent with previous studies (Akçay \& Hazeltine, 2007, 2008; Egner et al., 2007; Funes et al., 2010; Notebaert \& Verguts, 2008; Wendt et al., 2006). That is, control does not appear to be global but instead resides at the set-level. Even though both tasks used the same S-R mapping rules and both tasks involved conflict from irrelevant information that was identical to the relevant stimulus except for an earlier onset, conflict on one task did

\footnotetext{
${ }^{1}$ Because these categories of trials are overlapping, they are not summed to determine the total proportion of excluded trials. The percentages reported in the text are derived from the analysis of the data and deviate only slightly from what would be expected by chance $(25 \%)$.
} 
not produce sequential modulations on the other. We examine the nature of the set-level control in the subsequent experiments.

No other main effects or interactions were significant.

Item-level sequential modulations. Although the previous analysis provided strong evidence for set-level control, it remains possible that item-based control also occurred, even if it was not responsible for the sequential modulation. To test this, we performed a three-way ANOVA, with previous congruency, current congruency, and flanker history as factors. As in the analysis of set-level sequential modulations, we removed all types of stimulus repetitions (e.g., trials in which the target or flanker repeated and trials in which the target became the flanker or vice versa). We removed these trials for two reasons: First, we wished to make the analysis as analogous as possible to that used to identify set-level sequential modulations. Second, we wished to differentiate between item-level control effects and stimulus repetition effects.

Flanker history indicates whether the flanker on the current trial was a target or a flanker in an incongruent trial the last time it appeared. Because the same stimulus appeared as the target and flanker on congruent trials, we did not differentiate between incongruent target trials and congruent target trials. ${ }^{2}$ We collapsed across modalities and modality-switches to increase the number of observations in each cell and because the flanker history factor always referred to a trial that had occurred at least two switches ago, given that all immediate repetitions were eliminated.

The flanker history $\times$ previous congruency $\times$ current congruency ANOVA revealed a significant main effect of flanker history, $F(1,15)=12.93, p<.005, M S E=362.97$. Participants were 12 ms faster when the flanker on the present trial last appeared as a target $(555 \mathrm{~ms})$ than when it last appeared as an incongruent flanker $(567 \mathrm{~ms})$. Moreover, flanker history interacted with congruency, $F(1,15)=5.00, p<.05, M S E=1295.94$; that is, the congruency effect was $28 \mathrm{~ms}$ larger when the flanker on the current trial was a target the last time it appeared $(165 \mathrm{~ms})$ than when the flanker was an incongruent flanker the last time it appeared (137 ms).

This finding might initially suggest evidence for item-specific control. However, as depicted in Figure 2, the interaction reflects a change only in the RTs of congruent trials. On these trials, the

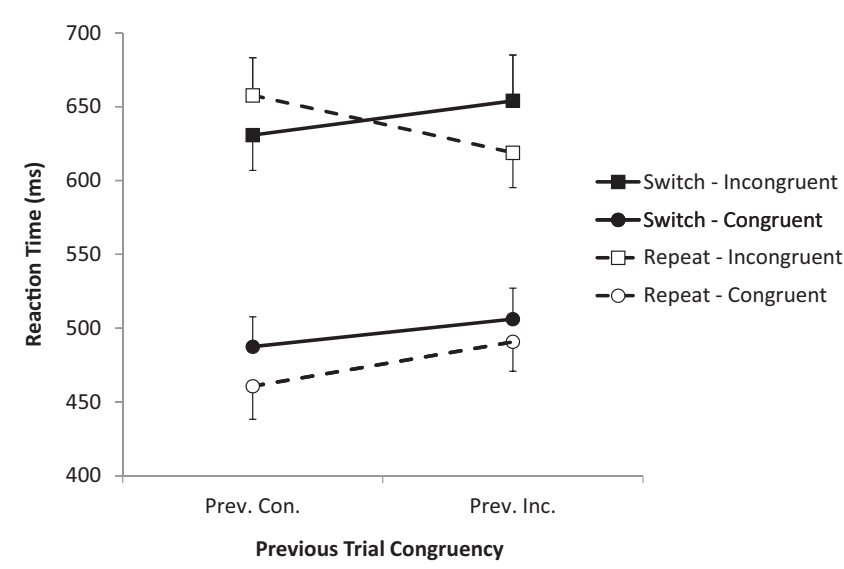

Figure 1. Reaction times from Experiment 1 for the switch (filled) and repeat (unfilled) task trials plotted with the congruency of the previous trial on the $x$-axis.

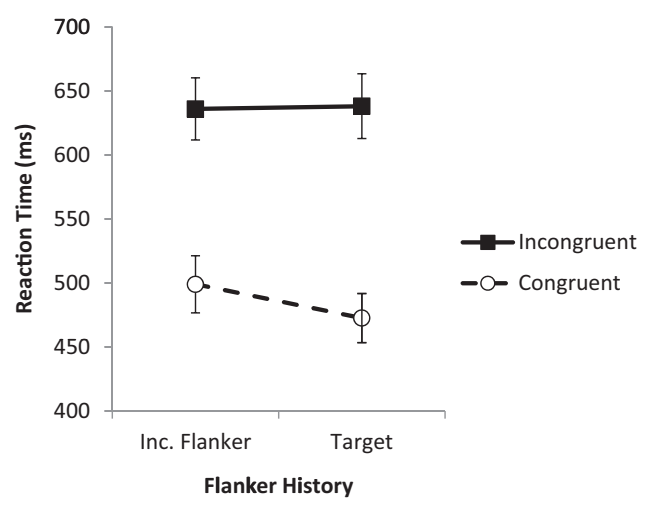

Figure 2. Reaction times from Experiment 1 for congruent (open circles) and incongruent (filled squares) trials plotted with the history of the flanker on the $x$-axis.

target and the flanker are identical, so the flanker history variable indicates the history of the target as well. In this light, the differences in the congruency effect as a function of flanker (and target) history are more consistent with negative priming (e.g., Milliken et al., 1998; Tipper \& Cranston, 1985). On congruent trials, responses were produced $26 \mathrm{~ms}$ faster, $t(15)=5.25, p<.0001$, when the target was previously a target than when it was previously an incongruent flanker. On incongruent trials, previously congruent and previously incongruent flankers produced RTs within $2 \mathrm{~ms}$ of each other, $t<1$. It is unclear why item-specific control, if it was engaged, would affect congruent and not incongruent trials. Thus, the results suggest that negative priming may provide a source of sequential modulations that are independent of the control processes implemented at the set-level.

All trials in which an item appeared, either as the flanker or as the target, on consecutive trials were eliminated from the analysis, so the minimum distance between a given flanker and the last time that item appeared either as a flanker or a target was at least three trials, given that a task switch occurred every other trial. Thus, the effect of flanker history on congruent trials speaks to the persistence of the negative priming effect across trials in four-choice tasks in which the same stimuli appear frequently.

The interaction between current congruency, previous congruency, and flanker history was not significant, $F<1$, indicating that the task-specific sequential modulations did not stem from inhibition of specific items. To confirm this conclusion, we performed separate $t$ tests on the congruency effects for previously incongruent versus previously congruent trials across the four combinations of switch and flanker history. For both levels of flanker history (flanker previously congruent, flanker previously incongruent), the difference in the congruency effect was significantly larger when the previous trial was congruent than when it was incongruent for repeated task trials, $t$ 's $>3.11$, $p$ 's $<.01$, but not for switch trials, $t$ 's $<1.6, p$ 's $>$.1. In other words, sequential modulations were

\footnotetext{
${ }^{2}$ We performed a supplemental analysis to examine whether flankers that had previously been targets on congruent trials and flankers that had previously been targets on incongruent trials produced different patterns of results. Making this distinction did not affect the basic pattern of findings reported in the analysis lumping these two types of trials together.
} 
consistently observed across different flanker histories, confirming that they did not stem from item-specific changes in processing. All other effects and interactions were described in the analysis of set-level modulations.

It is possible that target history also plays a role in the flanker effects and that its impact has masked the effects of flanker history. Unfortunately, it is not possible to add target history into a factorial design, because the target and flanker are identical on congruent trials. ${ }^{3}$ However, it is possible to factorially analyze target and flanker history considering only the incongruent trials, which are the critical ones. The results of this $2 \times 2$ ANOVA considering only incongruent trials (without any repetitions) show no indication of any main effect or interaction, all $F \mathrm{~s}<1$. In sum, there was no evidence for item-level sequential modulations beyond negative priming.

Persistence of set-level modulations. There are two classes of explanation for why switches eliminate sequential modulations. One account is that the switches simply reset all control parameters. In this case, there may only be one set of control parameters, as in global accounts of control, but they are reset when a switch occurs, so sequential modulations are only observed when there is no switch. The alternative account is that each set maintains its own control parameters and changes to these parameters do not affect processing of items from the other set. In this case, separate (local) control parameters must be maintained for the two sets of stimuli.

These two classes of accounts make distinct predictions on modality switch trials. According to the resetting account, the congruency effect on a modality switch trial should not be moderated by any of the previous trials, whereas, according to the local control parameter account, the congruency effect may depend on the congruency of the last trial in which the stimuli belonged to the same set. Akçay and Hazeltine (2008) tested these predictions using two Simon tasks and found that, on switch trials, the congruency of the last trial of the same set affected the magnitude of the congruency effect. Thus, they concluded that control involved changes in task-specific parameters and that switching did not result in a global resetting of task parameters. However, in that study, all stimuli were visual, and they did not rule out the possibility that sequential modulations were coming from itembased control. Therefore, we performed a two-way ANOVA examining only the switch trials and collapsing across modality and flanker history. Current congruency and last modality congruency (i.e., the congruency of the $n-3$ trial, which was the last trial of the same modality as the current trial) were factors. To make this analysis comparable to the first analysis of set-level sequential modulations, we eliminated all repetitions from the $n-3$ trials. That is, any trial in which the target or flanker from the last trial of the same modality appeared as either the target or flanker was removed, so that feature repetition (across the intervening task) effects were not confound with set-level sequential modulations.

There was a main effect of current congruency, $F(1,15)=$ 224.02, $p<.0001, M S E=1193.73$, a marginal effect of last modality congruency, $F(1,15)=3.57, p=.08, M S E=386.13$, and a nonsignificant trend toward an interaction, $F(1,15)=1.88$, $p=.19, M S E=877.66$. The trend toward an interaction reflected that when the last trial of the same modality was congruent, the congruency effect was $139 \mathrm{~ms}$ (492 vs. $631 \mathrm{~ms}$ ), but when the last trial of the same modality was incongruent, the congruency effect was $119 \mathrm{~ms}$ (493 vs. $612 \mathrm{~ms}$ ). In other words, the congruency of the trial of the same modality, performed three trials previously, may have moderated the magnitude of the congruency effect on the present trial just as the congruency of the previous trial affected the magnitude of the congruency effect on modality repeat trials. We take this issue up in the subsequent experiments.

Accuracy. The accuracy data were submitted to identical ANOVAs as the RT data. For the set-level ANOVA, only the main effect of congruency, $F(1,15)=12.82, p<.005, M S E=.001$, and the interaction between modality and switch, $F(1,15)=$ $11.41, p<.005, M S E=.001$, were significant. Participants were $1 \%(98 \%$ vs. $97 \%)$ more accurate when the current trial was congruent than when it was incongruent. They were also more accurate on auditory trials when the modality switched (99\% vs. 97\%), but more accurate on visual trial when the modality repeated (98\% vs. 97\%). We have no explanation for this small effect. For the item-level ANOVA, only the main effect of current congruency was significant, $F(1,15)=9.57, p<.01, M S E=.0001$ (see above). For the set-persistence ANOVA, none of the main effects or interactions was significant. In short, there was no evidence that speed-accuracy trade-offs were mediating any of the reported effects in the RT data.

To summarize, the findings from Experiment 1 indicate that sequential modulations are robust-and in the traditional direction-in the temporal flanker task. The modulations are specific to the set-level but not to individual items. These results rule out a host of potential explanations. For example, unlike previous demonstrations of local sequential modulations (e.g., Akçay \& Hazeltine, 2007, 2008; Egner et al., 2007; Funes et al., 2010; Notebaert \& Verguts, 2008; Wendt et al., 2006), the two tasks used in the present experiment involved the same rules (i.e., respond to the identity of the second item while ignoring the identity of the first) and the same stimuli for relevant and irrelevant information. Thus, they do not appear to arise from residual attention settings from one trial to the next, unless task-specific contextual parameters such as "first item" and "second item" are included in the attention settings.

An alternative account of these findings is that sequential modulations result from modality-specific adjustments in perceptual processing; ${ }^{4}$ for example, participants might avert their eyes slightly and return them to the center of the screen after the flanker but before the target. This strategy would minimize sequential modulations for visual trials but not auditory trials, but some analogous strategy might be possible for auditory trials. If participants always adopted one of these strategies for the current trial

\footnotetext{
${ }^{3}$ We are grateful to Mike Wendt for pointing out the possible role of target history. It is possible to do an ANOVA replacing the flanker history factor with a target history factor. The results of this analysis reveal no main effect of target history and only one significant interaction involving the factor: target history $\times$ congruency, $F(1,15)=10.44, p<.01, M S E=$ 800.43. As with flanker history, congruent trials were performed faster when the target had previous appeared as a target $(471 \mathrm{~ms})$ than when it had appeared as a flanker (495 ms), but there was no discernable effect of target history on incongruent trials (target: $639 \mathrm{~ms}$, flanker: $643 \mathrm{~ms}$ ). Thus, the results are striking consistent with those from the analysis of flanker history.

${ }^{4}$ We are grateful to Mike Wendt for suggesting this possible explanation.
} 
based on the modality of the previous trial, then modality-specific sequential modulations would be observed. We test this account in the subsequent experiments.

\section{Experiment 2}

The results from Experiment 1 indicate that sequential modulations of congruency effects are local to the set-level. The two stimulus sets used in Experiment 1, while using the same S-R mapping at a conceptual level, were distinct in the sense that the stimuli were presented along separate modalities, vision and audition. This raises a question of how distinct the sets need be to produce local sequential modulations. For example, in most studies examining local versus global control processes, both sources of irrelevant information are visual but exist along distinct dimensions, such as color and space. This has led researchers to conclude that control processes act on specific stimulus dimensions, such as shape and location (see Egner, 2008; Notebaert \& Verguts, 2008). An alternative proposal offered by Funes and colleagues (2010) is that control processes are bounded by the type of conflict, defined in terms of dimensional overlap (see Kornblum et al., 1990). Both of these accounts easily accommodate the data from Experiment 1, given that the two stimulus sets involved distinct modalities.

We further tested the boundary conditions for control processes in Experiment 2 by using two sets of visual stimuli, one identical to the visual stimuli used in Experiment 1 and the other based on spatial information, as in other studies finding local control (e.g., Akçay \& Hazeltine, 2007; Egner et al., 2007; Funes et al., 2010; Notebaert \& Verguts, 2008; Wendt et al., 2006). The purpose of this manipulation was to determine whether distinct sets of stimuli, one relying on shape information and one relying on spatial information, were sufficient to produce set-level control effects in the temporal flanker task, as in previous studies using distinct dimensions of visual stimuli in other conflict inducing tasks (e.g., Simon, Stroop, and Eriksen flanker tasks).

\section{Method}

Participants. Sixteen undergraduate students from Georgia Institute of Technology with ages ranging from 18-22 completed the study to fulfill a requirement for a psychology course. Four of the participants were female. Each participant completed two sessions of the experiment. One of the participants was eliminated from the analysis because they responded incorrectly on more than $10 \%$ of the trials. One participant was eliminated from the analyses for making errors on more than $10 \%$ of trials.

Apparatus and stimuli. The apparatus and stimuli were identical to those used in Experiment 1, except that the auditory stimuli were replaced by visuospatial stimuli. The spatial stimuli were a horizontal row of four white dashes presented just below fixation centered about the vertical midline. Each dash was $1.5 \mathrm{~cm}$ long and they were separated by $1 \mathrm{~cm}$. Above one of the four dashes, a filled white circle (diameter $=1.5 \mathrm{~cm}$ ) appeared on each trial. The location of the circle indicated which key to press in a spatially compatible fashion. Participants pressed the ' $v$ ' key with their right index fingers when they heard or saw ' $A$ ' or the leftmost circle, the ' $b$ ' key with the right middle fingers when they saw ' $B$ ' or the circle second from the left, and so forth The duration of the spatial stimuli was identical to the duration of the letters; both the flanker and target were presented for $250 \mathrm{~ms}$, and they were separated by a $100 \mathrm{~ms}$ ISI.

All other aspects of the experiment, including the timing of the events, were identical to Experiment 1.

\section{Results and Discussion}

Reaction time. Trials with incorrect responses (2\%), following incorrect responses, less than $200 \mathrm{~ms}(<1 \%)$, or greater than $2500 \mathrm{~ms}(1 \%)$ were removed from the RT analysis. The data were trimmed as in Experiment 1 so that trials in which either a target or flanker from the previous trial appeared as either a target or flanker in the current trial were eliminated. We also eliminated response repetitions because eliminating target repetition removed the response repetitions from the task repeat trials. The data were submitted to the same three ANOVAs as in Experiment 1.

Set-level sequential modulations. The task $\times$ switch $\times$ previous congruency $\times$ current congruency ANOVA revealed significant main effects of task, $F(1,14)=48.46, p<.0001$, $M S E=4080.57$, previous congruency, $F(1,14)=6.29, p<.05$, $M S E=685.23$, and current congruency, $F(1,14)=218.05, p<$ $.0001, M S E=4121.98$. The main effect of task indicated that the spatial stimuli were responded to faster than the letter stimuli (455 vs. $513 \mathrm{~ms}$ ), and the main effect of previous congruency replicated the finding from Experiment 1 and indicated that trials following congruent trials $(480 \mathrm{~ms}$ ) were performed faster than trials following incongruent trials ( $488 \mathrm{~ms}$ ). The effect of congruency indicated a robust flanker effect, with congruent trials being performed faster than incongruent trials (424 vs. $544 \mathrm{~ms}$ ). This effect interacted with task, $F(1,14)=71.54, p<.0001, M S E=1038.68$, with the congruency effect being larger for letter trials (436 vs. $591 \mathrm{~ms}$ ) than for spatial trials (412 vs. $497 \mathrm{~ms}$ ).

Of critical interest were the sequential modulations. Previous congruency and current congruency interacted in the typical fashion, $F(1,14)=8.79, p<.05, M S E=459.88$, indicating that the congruency effect was larger after congruent trials (416 vs. 544 ms) than after incongruent trials (432 vs. 544 vs. ms; see Figure 3 ). However, the interaction between these two factors was not moderated by any higher level interactions, all $F_{\mathrm{s}}<1$. In particular,

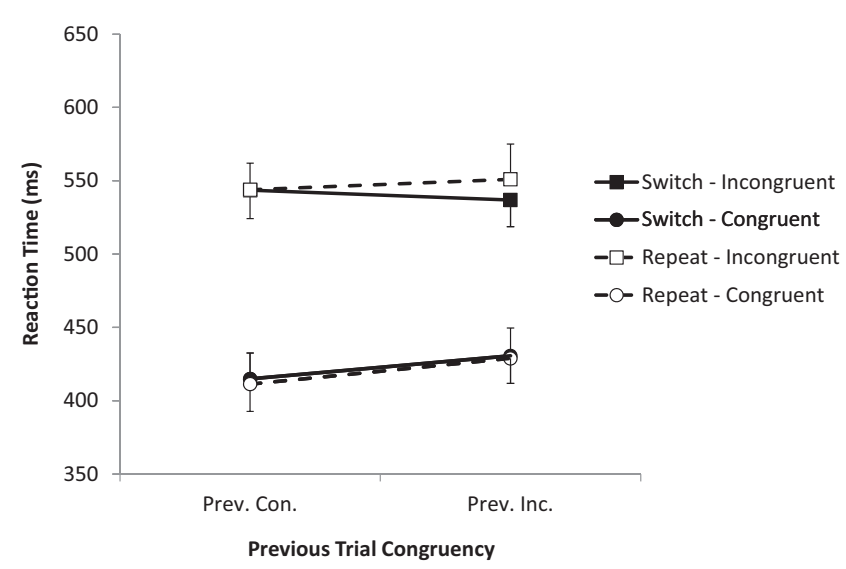

Figure 3. Reaction times from Experiment 2 for the switch (filled) and repeat (unfilled) task trials plotted with the congruency of the previous trial on the $x$-axis. 
there was no indication that the sequential modulation was smaller on switch trials (129 vs. $106 \mathrm{~ms}$ ) than on repeated task trials (133 vs. $122 \mathrm{~ms}$ ).

Thus, in contrast to Experiment 1, there was no evidence that sequential modulations were confined to within the two stimulus sets. Despite the fact that one set of stimuli was based on shape (letter) and the other location, global control was observed.

No other main effect or interaction was statistically significant.

Item-level sequential modulations. The flanker history $\times$ previous congruency $\times$ current congruency ANOVA revealed no main effects or interactions not described in the previous analysis. However, the main effect of flanker history, $F(1,14)=4.11, p=$ $.06 ; M S E=264.31$, and the flanker history $\times$ current congruency interaction, $F(1,14)=2.95, p=.11, M S E=232.78$, both approached significance, nearly replicating the findings from Experiment 1. The pattern was identical to that of Experiment 1: incongruent trials showed no effect of flanker history (548 vs. 549 $\mathrm{ms}, t<1$ ), but congruent trials, for which the flanker history variable also described the history of the target, produced faster responses when the flanker/target was previously a target (418 ms) than when the flanker/target was previously a flanker $(429 \mathrm{~ms})$, $t(14)=4.78, p<.0005$. Thus, the findings essentially replicate those of the previous experiment; there is evidence for negative priming but no indication that the history of the flanker determines the sequential modulation.

As in Experiment 1, we performed an ANOVA on the incongruent trials (trimmed of all stimulus repetitions) with both target history and flanker history as factors to confirm that the target's history was not masking any latent effect of flanker history on these trials. There were no main effects nor was the interaction significant, all $F_{\mathrm{s}}<1$. These findings confirm that the history of the particular flanker on the current trial has little effect on the congruency effect.

Persistence of set-level modulations. We performed an ANOVA restricted to switch trials to examine the effects of previous congruency, current congruency, and the congruency of the last trial of the same task (i.e., the n-3 trial). As in Experiment 1 , we eliminated all trials in which the target or flanker appeared, either as a target or a flanker, in the n-3 trial. There was a significant interaction between current congruency and last task congruency, $F(1,14)=14.32, p<.005, M S E=338.02$. When the last trial involving the same task was congruent, the congruency effect was $124 \mathrm{~ms}$ ( $419 \mathrm{~ms}$ vs. $543 \mathrm{~ms}$ ), whereas when the last trial involving the same task was incongruent, the congruency effect was $99 \mathrm{~ms}$ (431 ms vs. $530 \mathrm{~ms}$ ). This pattern suggests that, even though sequential modulations crossed the task boundary, there was an additional modulatory effect from the last trial of the same task. $^{5}$

Only current congruency, $F(1,14)=133.12, p<.0001, M S E=$ 2805.17, and the interaction between current congruency and previous congruency, $F(1,14)=10.80, p<.01, M S E=844.31$, were also significant. These effects have been discussed previously.

Accuracy. The accuracy data were again submitted to identical ANOVAs as the RT data. For the set-level ANOVA, only the main effects of switch, $F(1,14)=5.86, p<.05, M S E=.0001$, and congruency, $F(1,14)=6.29, p<.05, M S E=.003$, were significant. Participants were $1 \%(99 \%$ vs. $98 \%)$ more accurate when the trial repeated the task than when it switched and $1 \%$
(99\% vs. 98\%) more accurate when the current trial was congruent than when it was incongruent. No interactions were significant. For the item-level ANOVA, only the main effect of current congruency was significant, $F(1,14)=6.04, p<.05, M S E=.001$ (see above). For the set-persistence ANOVA, there was interaction between previous congruency and current congruency, $F(1,14)=$ 20.17, $p<.001, M S E=.0001$, indicating that performance advantage for congruent trials was greater following congruent trials that following incongruent trials, consistent with the RT data. The congruency of the last trial of the same modality produced no main effect or interactions. Thus, there was no evidence that set-level control or speed-accuracy trade-offs were mediating any of the reported effects in the RT data.

In sum, the pattern of results was quite distinct from that of Experiment 1. In that experiment, sequential modulations were confined to within a task, whereas in Experiment 2, sequential modulations crossed tasks. The only difference between the two experiments was the relationship between the two tasks: In Experiment 1 , the stimuli occurred along distinct modalities but were conceptually related, whereas in Experiment 2, all the stimuli were visual but were conceptually unrelated. Given that the two sets of stimuli in Experiment 2 were based on different stimulus dimensions - the letters were based on shape information and the spatial stimuli were based on local information-it does not appear that this factor determines whether sequential modulations cross from one set of stimuli to another (see Egner, 2008; Funes et al., 2010).

What does determine the boundaries of control processes? One obvious possible answer is that stimulus modalities provide the critical boundaries. This might reflect modality specific control processes-that is, that there is a control process for visual stimuli and a distinct control process for auditory stimuli (see Schumacher, Schwarb, Lightman, \& Hazeltine, in press). Alternatively, it might reflect modality-specific strategies, such as averting the eyes for the first stimulus, which would have little effect when the next trial involved auditory stimuli. However, this interpretation is not consistent with other studies that have used only visual stimuli and found local control (e.g., Akçay \& Hazeltine, 2008, 2011; Egner et al., 2007; Funes et al., 2010; Notebaert \& Verguts, 2008). Another possibility is that for both the letter and spatial stimuli, the source of interference was essentially the same: irrelevant information presented just before the relevant information. If control processes were bounded by the source (e.g., first vs. second stimulus) rather than the relevant stimulus dimension (e.g., shape or location), then sequential modulations would be expected to cross the stimulus sets. A limitation for this account is that it does not provide an obvious account for the pattern of data from Experiment 1, but modality might also play a role. A third possibility is that the salience of the stimulus sets determines whether control is local, and, given that both sets required the same responses, the difference between these two visual sets used in Experiment 2 were insufficient to instantiate boundaries for control processes. Ac-

\footnotetext{
5 This finding was unexpected given the robust modulation based on the congruency of the previous trial. To verify that there was not a general modulatory effect based on the n-3 trial regardless of the task relationship, we performed an analogous ANOVA on Experiments 1-3 on the task repeat trials. In each experiment, there was no indication of any effect of the n-3 congruency on the task repeat trials, all $F_{\mathrm{s}}<1$.
} 
cording to this explanation, the boundaries are flexible and based on task demands rather than the stimulus features/dimensions. These possibilities are investigated in Experiment 3.

\section{Experiment 3}

The results of Experiment 2 were somewhat unexpected. ${ }^{6}$ While the two stimulus sets, the letters and the spatial stimuli, were easily separable, the congruency of the previous trial had the same effect on performance for switch and task-repeat trials-that is, control appeared to take place at a global level. As noted above, this finding stands in contrast to previous studies that have used only visual stimuli and found local control.

In many of the previous studies reporting local control, the two sources of irrelevant information involved distinct sources. For example, in the Egner, Delano, and Hirsch (2007) study, Stroop interference did not produce sequential modulations of Simon interference and vice versa, and in the Funes et al. (2010) study, Stroop interference did not produce sequential modulations of flanker interference and vice versa (see also, Akçay \& Hazeltine, 2011). In contrast, while Experiment 2 used spatial and letter stimuli, the source of the interference was always the first item. Thus, one could argue that the source of the interference was the same across the two tasks, leading to global control. This explanation does not account for the data from Experiment 1, but it could also be the case that the distinct stimulus modalities necessarily engage distinct control processes.

One way to address this issue is to attempt to determine what constitutes the boundaries of control processes by systematically varying the sources of the relevant and irrelevant information. This would entail testing a range of types of conflict (e.g., Stroop, flanker, Simon) across the visual and auditory modalities (at least) and identifying the critical factors that lead to set-level sequential modulations. For example, are the same control processes engaged by irrelevant arrow flankers and irrelevant location information (both of which involve Simon interference)? However, a limitation of this approach is that it is unlikely to be exhaustive. That is, it may be very difficult to establish that the identified set of control processes is complete, and the domains of each will be hard to define.

An alternative approach is to examine the task factors that can affect the boundaries of control processes. This was our motivation for Experiment 3. Rather than manipulating the stimuli to identify the critical differences required to produce set-level sequential modulations, we examined whether factors beyond stimulus properties could affect the scope of the sequential modulations. That is, we examined whether stimulus properties alone determine whether control is local or global. Like the former approach, this strategy does not readily provide an exhaustive set of criteria for establishing the boundaries of control mechanisms. However, it does determine whether the locus of control resides in feature-based perceptual systems or more flexible higher-level systems (see Egner, 2008).

Along these lines, Akçay and Hazeltine (2008) found that sequential modulations were not observed from one Simon task to another Simon task when the two tasks were clearly distinct and required responses with different hands. Given that both Simon tasks involved the suppression of spatial information, this finding suggests that control processes may not be bounded by the stim- ulus dimension. Instead, it indicates that the task demands may play a role in shaping the boundaries of control. When a particular feature is salient and task relevant, it may form a boundary for control processes. When the same feature is irrelevant to the task, global control may be observed.

To test this, we used the same stimuli that produced global sequential modulations in Experiment 2 and changed the task demands to emphasize the distinction between the two sets. The change was restricted to the responses: when letters were presented, participants responded with their left hands, and when spatial stimuli were presented, participants responded with their right hands. If the locus of control is determined strictly by stimulus properties, then the results should be identical to Experiment 2. However, if the locus of control can be affected by changes in task demands-that is, the requirement to respond based on the set to which the current stimulus belongs- then local sequential modulations may be observed.

\section{Method}

Participants. Sixteen undergraduate students from Georgia Institute of Technology with ages ranging from 18-28 completed the study to fulfill a requirement for a psychology course. Six of the participants were female. Each participant completed two sessions of the experiment. One participant was eliminated from the analyses for making errors on more than $10 \%$ of trials.

Apparatus and stimuli. The apparatus and stimuli were identical to those used in Experiment 2, except that the letter stimuli were responded to with the four fingers of the left hand using the keys 'a,' 's,' 'd', and 'f,' and the spatial stimuli were responded to with the four fingers of the right hand using the keys ' $h$,' 'j,' ' $k$,' and 'l.' All other aspects of the experiment, including the timing of the events, were identical to Experiment 2.

\section{Results and Discussion}

Reaction time. Trials with incorrect responses (3\%), following incorrect responses, less than $200 \mathrm{~ms}(<1 \%)$, or greater than $2500 \mathrm{~ms}(1 \%)$ were removed from the RT analysis. The data were trimmed and submitted to three ANOVAs as in Experiments 1 and 2 .

Set-level sequential modulations. The task $\times$ switch $\times$ previous congruency $\times$ current congruency ANOVA revealed significant main effects of task, $F(1,14)=65.87, p<.0001$, $M S E=5597.63$, switch, $F(1,14)=9.64, p<.01, M S E=669.90$, and current congruency, $F(1,14)=109.52, p<.0001, M S E=$ 4157.77. Again, the spatial stimuli were performed faster than the letter stimuli (467 vs. 538 ms). However, unlike Experiment 2 but like Experiment 1, switch trials (506 ms) were performed more slowly than repeated task trials (499 ms), although the magnitude of the effect was small. The effect of congruency indicated a robust flanker effect, with congruent trials being performed faster than incongruent trials (472 vs. $533 \mathrm{~ms}$ ). This effect interacted with task, $F(1,14)=39.20, p<.0001, M S E=1962.70$, as it did in

\footnotetext{
${ }^{6}$ We have replicated the finding of global control with other combinations of visual stimulus sets, including animals pictures paired with visual letters and animal pictures paired with tool pictures, so we are confident that the results of Experiment 2 are not anomalous.
} 
Experiment 2, with the congruency effect again being larger for letter trials (490 vs. $586 \mathrm{~ms}$ ) than for spatial trials (454 vs. $480 \mathrm{~ms}$ ). Task also interacted with switch, $F(1,14)=13.67, p<.005$, $M S E=498.60$, with switch costs being larger for letter task (547 vs. $530 \mathrm{~ms}$ ) than for the spatial task (466 vs. $468 \mathrm{~ms}$ ).

As in both previous experiments, there was a significant interaction between previous congruency and current congruency, $F(1$, $14)=21.18, p<.0001, M S E=475.60$, with the congruency effect being larger after congruent trials ( $474 \mathrm{vs} .551 \mathrm{~ms}$ ) than after incongruent trials (470 vs. $515 \mathrm{~ms}$ ). However, like Experiment 1 but unlike Experiment 2, the sequential modulation was itself moderated by switches, as indicated by the three-way interaction between previous congruency, current congruency, and switch, $F(1,14)=39.20, p<.0001, M S E=1962.70$. The interaction, depicted in Figure 4, reflects the fact the sequential modulation was not observed following switch trials ( 89 vs. $81 \mathrm{~ms}, t<1)$ but was observed following repeat trials $(113$ vs. $69 \mathrm{~ms}, t(14)=5.35$, $p<.0005)$.

To confirm that the change in the manual responses affected the pattern of sequential modulations, we performed a follow-up analysis combining the data from Experiments 2 and 3 and using experiment as a between subjects factor. This procedure violates the assumption of random assignment, because Experiment 3 was begun after Experiment 2 was completed, but is otherwise justified given the strong similarity between the two experiments.

The main effect of experiment was not significant, $F<1$, indicating that having to make responses with either hand did not dramatically affect RT. However, the experiment factor did interact with switch, $F(1,28)=6.70, p<.05, M S E=709.11$, and with current congruency, $F(1,28)=8.39, p<.01, M S E=4189.88$. Small switch costs were observed for Experiment 3 (499 vs. 506 $\mathrm{ms}$ ) but not in Experiment 2 (485 vs. $483 \mathrm{~ms}$ ). Congruency effects, on the other hand, were larger for Experiment 2 (424 vs. $544 \mathrm{~ms}$ ) than for Experiment 3 (472 vs. $533 \mathrm{~ms}$ ).

Most critically, there was a four-way interaction between experiment, switch, previous congruency, and current congruency, $F(1,28)=4.79, p<.05, M S E=877.36$. Participants in Experiment 2, who responded with the same hand to both the spatial and letter stimuli, showed the same sequential modulations on switches

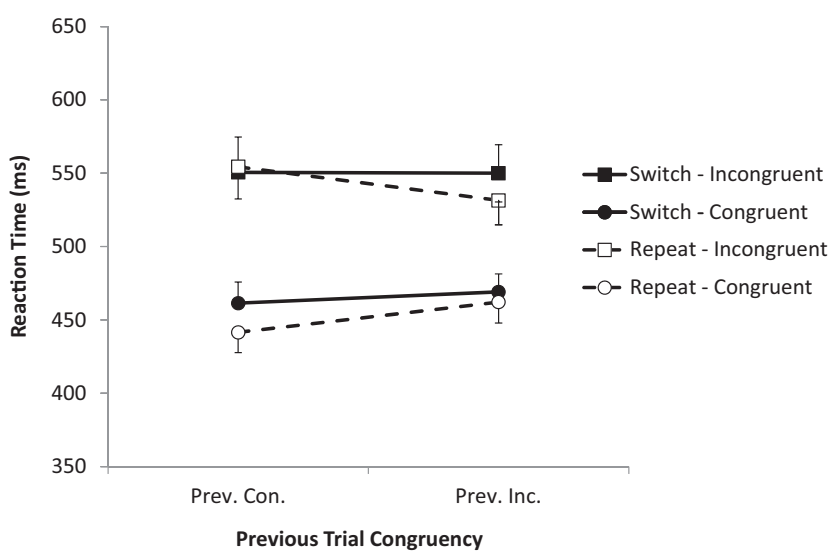

Figure 4. Reaction times from Experiment 3 for the switch (filled) and repeat (unfilled) task trials plotted with the congruency of the previous trial on the $x$-axis. and repeat task trials. In contrast, participants in Experiment 3, who responded with the left hand to letter stimuli and the right hand to spatial stimuli, showed sequential modulations on repeat task trials but not on switches. In short, Experiment 2 produced global control and Experiment 3 produced local control.

These findings demonstrate that the scope of sequential modulations does not depend on stimulus properties alone. Rather, response properties can determine the scope of sequential modulations as well (see Akçay \& Hazeltine, 2008). One might argue that each hand has its own control parameters, just as one might interpret the data from Experiment 1 as indicating that each stimulus modality has its own response parameters. The stimulus modality hypothesis is directly tested in Experiment 4. With regard to the within-hand control hypothesis, it appears unlikely because previous studies have reported sequential modulations in twochoice tasks with bimanual responses (e.g., Gratton et al., 1992; Stürmer et al., 2002) and one study reported sequential modulations from vocal to manual responses (Fernandez-Duque \& Knight, 2008). ${ }^{7}$ Thus, the data appear most consistent with the hypothesis that the boundaries of control processes are flexible and depend on the particular task demands.

There were no other significant interactions involving experiment, $F \mathrm{~s}<1$.

Item-level sequential modulations. As in Experiment 2, the flanker history $\times$ previous congruency $\times$ current congruency ANOVA was conducted examining only trials with no stimulus repetitions. It revealed no main effect or interactions not described in the previous analysis. However, the pattern was very consistent with the previous experiments: incongruent trials showed no effect of flanker history ( $545 \mathrm{vs} .547 \mathrm{~ms}, t<1$ ), but congruent trials, for which the flanker history variable also described the history of the target, produced faster responses when the flanker/target was previously a target $(454 \mathrm{~ms})$ than when the flanker/target was previously a flanker $(471 \mathrm{~ms}), t(14)=3.46, p<.005$. A follow-up ANOVA on the incongruent trials without stimulus repetitions that included flanker history and target history revealed no significant main effects or interactions, all $F_{\mathrm{S}}<1$. As in the previous experiments, there was clear evidence for negative priming, but no indication that control is implemented at the item-level.

Persistence of set-level modulations. The ANOVA restricted to switch trials and examining the effects of previous congruency, current congruency, and the congruency of the last trial of the same task produced no significant effects beyond those described above. As in Experiments 1 and 2, stimulus repetitions of all kinds were removed to isolate the effects of persistent set-level modulations. The ANOVA reveal a significant main effect of current congruency, $F(1,14)=45.15, p<.0001 ; M S E=$ 5063.56 and a significant interaction between current congruency and the congruency of the last trial of the same task, $F(1,14)=$ $7.45, p<.05 ; M S E=971.78$. No other effects or interactions were significant. As in the previous experiments, when the last trial of

\footnotetext{
${ }^{7} \mathrm{We}$ also performed a control experiment identical to Experiment 2 except that the left two key presses were made with the left hand and the right two key presses were made with the right hand. The results were essentially identical to those of Experiment 2, with sequential modulations being observed when the previous trial required a key press with a different hand than the current trial.
} 
the same task was congruent, the congruency of the current trial produced a larger effect $(103 \mathrm{~ms})$ than when the last trial of the same task was incongruent (72 ms).

This interaction, observed as a nonsignificant trend in Experiment 1 and a significant effect in Experiments 2 and 3, suggests that control processes do not alter global parameters such as the amount of resources devoted to the task or adjust the speedaccuracy trade-off in response to conflict. It appears that the two task sets maintain their own distinct control parameters that are reinstated once stimuli from that task reoccur. Thus, the absence of sequential effects observed on switch trials does not appear to stem from a global resetting of control parameters during the switch, consistent with Akçay and Hazeltine (2008). Note that the interaction cannot be explained in terms of item-based control, given that the previous task congruent trials and the previous task incongruent trials were composed equally of trials in which the flanker had previously been a target and trials in which the flanker had previous been an incongruent flanker. Therefore, control parameters appear to persist across switches, but they only affect processing within a task set, provided the distinction between the task sets is sufficiently salient.

Accuracy. The accuracy data were again submitted to identical ANOVAs as the RT data. For the set-level ANOVA, only the main effect of congruency, $F(1,14)=8.18, p<.05, M S E=.002$, was significant. Participants were $1 \%$ (96\% vs. 95\%) more accurate when the current trial was congruent than when it was incongruent. No interactions were significant. For the item-level ANOVA, previous congruency and current congruency interacted, $F(1,14)=7.88, p<.05, M S E=.0001$, indicating that performance advantage for congruent trials was greater following congruent trials that following incongruent trials, consistent with the RT data. For the set-persistence ANOVA, there was a significant interaction between previous task congruency and current congruency, $F(1,14)=4.87, p<.05, M S E=.001$, indicating that the benefit for congruent trials was larger when the last trial of the same modality was congruent (95\% vs. $98 \%$ ) than when the last trial of the same modality was incongruent ( $97 \%$ vs. $98 \%)$. That is, evidence for set-specific control parameters was obtained in the accuracy data. There was no evidence that speed-accuracy tradeoffs mediated any of the reported effects in the RT data.

In sum, Experiments 2 and 3 provide strong evidence that the boundaries of sequential modulations are flexibly determined based on the task demands; when the two sets of stimuli required overlapping responses, sequential modulations crossed the set boundary, and when they required distinct responses, the sequential modulations were specific to stimulus set. These findings are not easily accommodated by accounts that hold that sequential modulations stem from modality-specific strategies, because the stimuli in Experiments 2 and 3 were identical, yet distinct patterns of modulations were observed. For example, if participants were producing sequential modulations on visual trials by averting their eyes during the flanker presentation in Experiments 1 and 2, there is no reason not to expect the same pattern in Experiment 3. The fact that the responses determine whether or not sequential modulations cross tasks suggests that sequential modulations reflect changes at a post-perceptual level processing that is sensitive to the task demands. It is possible that participants alter the way they process the flankers based on the task demands, so that when the tasks require responses with the same hand, they change the way they process both visual and auditory stimuli after each trial; and when the tasks require responses with different hands, they change the way they process stimuli only along the present modality. However, this account is unparsimonious and, as we shall see, inconsistent with findings from Experiment 4. Thus, we propose that the most likely account of these data is that control is implemented at the level of the task set.

\section{Experiment 4}

The findings of Experiments 2 and 3 suggest that the relationship between stimulus dimensions and sequential modulations may be flexible. When differences between stimulus sets are taskirrelevant (i.e., when the stimulus set does not constrain which response should be produced), then control may be global. But, what, then, do we make of the data from Experiment 1, where the distinction between the visual and auditory stimuli was irrelevant for determining the response, yet the sequential modulations were modality-specific? One possibility is that stimulus modality imposes a hard boundary on control processes that is insensitive to the task demands. In other words, the proposal is that there is some flexibility within a stimulus modality regarding the boundaries of control, but stimuli in different modalities will always invoke distinct control processes.

Alternatively, it is possible that control processes are able to operate across stimulus modalities. According to this account, local control is observed when there is a boundary that causes the participants to conceptualize stimuli as belonging to separate tasks. In Experiment 1, the stimulus modality provided a salient cue that forms a boundary for sequential modulations even though it was task-irrelevant. In Experiment 2, the spatial and letter stimuli were never presented on the same trial, so there was no need to distinguish between these two sources of information. Moreover, because the two sets were both presented in the same modality, they were not sufficiently distinct to be encoded as separate. That is, participants could have encoded the two sets as a single task with an 8 stimulus to 4 response mapping. In Experiment 3, the stimulus set informed the participants which hand was required for the correct response, so the distinction between the sets became salient and provided a boundary for sequential modulations.

The two accounts differ primarily with regard to the explanation for the findings of Experiment 1. The modality account holds that local control was observed in Experiment 1 because the two sets involved distinct modalities, whereas the salience account holds that local control was observed because modality was a very salient source of variation among the stimuli. To examine whether it is salience rather than modality that plays the critical role, we tested whether sequential modulations could cross stimulus modalities when they provided a less salient distinction. It is unclear how to make the two modalities more similar, so we instead provided an alternative distinction (i.e., an additional source of variation) to compete with modality. We included two tasks, an animal task and a letter task, as well as two modalities, so that a given stimulus was either an animal or a letter and was either visual or auditory. We assumed that by providing a second means of categorizing the stimuli, we would make the stimulus modality distinction less salient, and create conditions under which global control might occur. Determining whether sequential modulations can cross over to different stimulus modalities will indicate 
whether the underlying control mechanisms operate within modality-specific processes or in amodal processes.

By varying both task and modality in Experiment 4, we expected that the modality changes would no longer provide strong category boundaries, especially because not every stimulus within a modality was conceptually related. Moreover, to increase the salience of task, we alternated tasks every other trial, and varied the modality pseudorandomly.

\section{Method}

Participants. Sixteen undergraduate students from Georgia Institute of Technology with ages ranging from 18-25 completed the study to fulfill a requirement for a psychology course. Seven of the participants were female. Two of the participants were eliminated from the analyses for making errors on more than $10 \%$ of the trials.

Apparatus and stimuli. The equipment was identical to that used in Experiment 1. There were two classes of stimuli, each associated with a task. The letter stimuli were identical to the visual and auditory stimuli used in Experiment 1. The visual animal stimuli were line drawings, white on black, of a dog, a cat, a bird, and a pig. Each drawing was $3 \mathrm{~cm}$ by $3 \mathrm{~cm}$ presented at fixation for $250 \mathrm{~ms}$. The auditory stimuli were .wav files of the words "dog", "cat", "bird" and "pig" spoken in a male voice at a comfortable volume. The files were edited so that each word lasted exactly $250 \mathrm{~ms}$, as in Experiment 1.

Procedure. Participants completed the experiment over two sessions on separate days. Each session began with two practice blocks, one with just the letter task and one with just the animal task. The stimulus modality of the trial was chosen randomly with the constraint that 30 visual trials and 30 auditory trials be presented on each 60 trial block. The remaining 10 blocks consisted of both tasks, switching every other trial (e.g., letter-letter-animalanimal. .). As in the single task blocks, the modality of the trial was random with the constraint that an equal number of visual and auditory trials be presented in each block. The mapping of stimuli to responses was identical to Experiments 1 and 2 for the letters. For the animals, participants pressed the ' $v$ ' key when a dog was presented, the ' $b$ ' key when a cat was presented, the ' $n$ ' key when a bird was presented, and the ' $m$ ' key when a pig was presented.

All other details of the experiment were identical to Experiments 1 and 2.

\section{Results and Discussion}

Reaction time. Trials with incorrect responses (5\%), following incorrect responses, less than $200 \mathrm{~ms}(<1 \%)$, or greater than $2500 \mathrm{~ms}(1 \%)$ were removed from the RT analysis. The first four blocks of each session were treated as practice and not analyzed. To examine sequential modulations, all trials in which the target repeated, the flanker repeated, the previous target became the flanker, the previous flanker became the target, or the correct response repeated were eliminated, as in the previous experiments, leaving $53 \%$ of the trials.

Set-level sequential modulations. The focus of this study was to compare the effects of modality switches and task switches. Therefore, in order to obtain sufficient observations per cell of the design, we collapsed across modality (i.e., whether the stimuli were visual or auditory) and task (i.e., whether the stimuli were letters or animals), and submitted the data to a four-way ANOVA with task-switch (i.e., whether the task on the previous trial was the same), modality-switch (i.e., whether stimulus modality on the previous trial was the same), previous congruency, and current congruency as factors.

The main effects of task-switch, $F(1,13)=11.34, p<.005$, $M S E=1311.03$ and congruency, $F(1,13)=214.84, p<.0001$, $M S E=4235.34$ were significant. The effect of task-switch reflects a $17 \mathrm{~ms}$ cost ( $722 \mathrm{~ms}$ vs. $705 \mathrm{~ms}$ ) for trials in which the task was different than on the previous trial. Although the modality switches were unpredictable (unlike the task switches), there was little evidence of any cost of switching modality, $F<1$ (716 ms vs. $711 \mathrm{~ms}$ ).

Modality-switch and task-switch did interact, $F(1,13)=7.84$, $p<.05, M S E=476.66$, as task-switch costs were larger when the modality remained the same (699 vs. $724 \mathrm{~ms})$ than when the modality also switched (711 vs. $720 \mathrm{~ms}$ ). Moreover, there was a three-way interaction between modality-switch, task-switch and congruency, $F(1,13)=8.12, p<.05, M S E=1275.47$, indicating that the congruency effect was larger when both task and modality repeated $(149 \mathrm{~ms})$ or switched $(133 \mathrm{~ms})$ than when task switched and modality repeated $(101 \mathrm{~ms})$ or task repeated and modality switched (127 ms).

Again, the focus of the analysis was on the previous congruency $\times$ current congruency interaction and any moderating factors. As in Experiments 1-3, the previous congruency $\times$ current congruency interaction was significant, $F(1,13)=19.52, p<.001$, $M S E=1490.60$, indicating that the congruency effect was larger after congruent trials $(150 \mathrm{~ms})$ than after incongruent trials (105 ms) (see Figure 5). Moreover, this interaction was not moderated by modality switches, task switches, or the combination of the two factors, all $F \mathrm{~s}<1$.

To confirm that the sequential modulations occurred across both modality switches and task switches in Experiment 4, we performed $t$ tests on the congruency effects after congruent and incongruent trials for modality-switch trials only. The congruency effect was significantly larger when the previous trial used a different modality and was congruent $(136 \mathrm{~ms})$ than when the previous trial used a different modality and was incongruent (97 $\mathrm{ms}), t(13)=3.13, p<.01$. There was also a significant sequential modulation when the task changed (164 vs. $112 \mathrm{~ms}, t(13)=3.29$, $p<.01)$. In fact, on the subset of trials when both the modality and task switched from the previous trial, the sequential modulation was significant (156 vs. $110 \mathrm{~ms}, t(13)=2.27, p<.05$ ).

Despite our efforts to make the task salient, there was no evidence that sequential modulations were sensitive to task boundaries. In many respects, this finding is analogous to the results of Experiment 2, in which two sets of stimuli were clearly distinct, yet the sequential modulations were global. It appears that the addition of the two tasks was sufficient to reduce the salience of the modalities so that sequential modulations crossed modality but insufficient to produce sequential modulations that were specific to task. Given that Experiment 3 demonstrated that sequential modulations can be local when both tasks are visual, we assume that the global modulations observed in Experiment 4 stem from the complexity of the task. When participants were confronted with two distinct sets of stimuli, each of which occurred along two stimulus modalities, the salience of all the boundaries was muted 

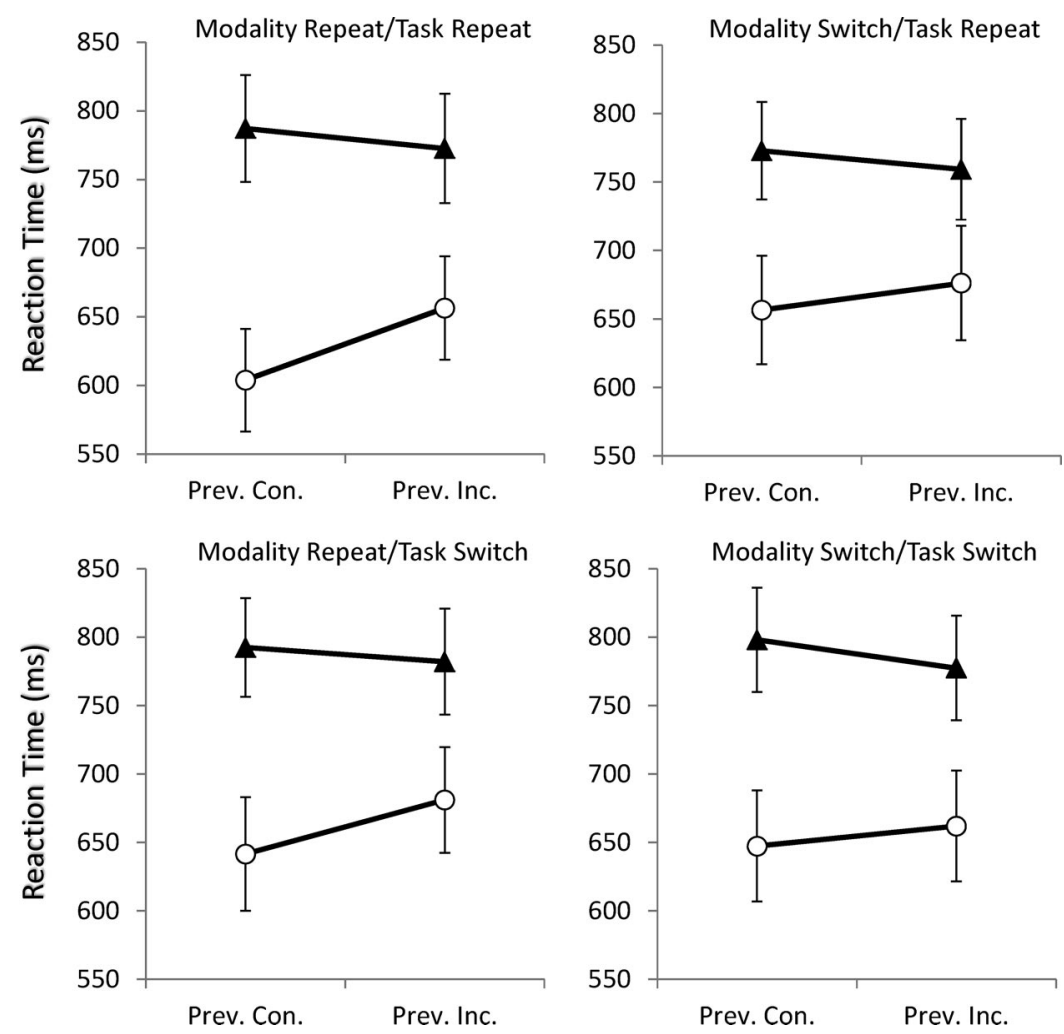

Figure 5. Reaction times from Experiment 4 for congruent (unfilled circles) and incongruent (filled triangles) trials plotted with the congruency of the previous trial on the $x$-axis. The four panels represent the four combinations of modality repeat/switch, organized in columns, and task repeat/switch, organized in rows.

and thus none affected control processes. Future work will examine what specific factors contribute to the instantiation of boundaries leading to local sequential modulations. For the present, we conclude that sequential modulations can cross stimulus modality under the appropriate task conditions and therefore likely reflect amodal control processes operating on post-perceptual representations.

No other main effects or interactions approached significance.

Item-level sequential modulations. Because there were 16 different stimuli used in Experiment 4, it was not possible to perform a meaningful analysis of item-level sequential modulations.

Persistence of set-level modulations. The ANOVA restricted to modality- and task-switch trials and examining the effects of current congruency, the congruency of the last trial of the same task, and the congruency of the last trial of the same modality produced no significant effects or interactions beyond the main effect of congruency, reported above. Although the power of this analysis was limited, these null results were expected given that there was little evidence that either task or modality imposed any boundaries on control processes.

Accuracy. The accuracy data were again submitted to identical ANOVAs as the RT data. For the set-level ANOVA, only the main effect of modality repetition was significant, $F(1,13)=5.79$, $p<.05, M S E=.001$, indicating that participants were $1 \%$ more accurate on modality repetitions $(96 \%)$ than on modality switches $(95 \%)$. There were no significant interactions. For the set- persistence ANOVA, there was a main effect of previous modality congruency, $F(1,13)=4.91, p<.05, M S E=.001$, indicating participants were more accurate when the last trial in the same modality was incongruent $(97 \%)$ than when it was congruent (95\%). We have no explanation for this effect, but there was no evidence of any interaction between either previous modality congruency or previous task congruency and current congruency, so there was no evidence of any modality- or task-specific control.

In sum, when the salience of modality was diminished by the presence of an additional form of variation (category), global control was observed. It appears that the modality-specific effects observed in Experiment 1 did not stem from hard-wired boundaries related to the neural specialization of the input systems (or downstream neural systems), but rather relate to the participants' organization of the task. Thus, we conclude that the control processes responsible for sequential modulations operate on high level representations that reflect the structure of the task, not the structure of the perceptual system.

\section{General Discussion}

The present experiments contribute three principal findings: (a) sequential modulations are observed when targets and flankers differ only with respect to their relative timing, (b) local sequential modulations occur at the set-level, and (c) the boundaries of sequential modulations are not determined only by stimulus properties. Each of these findings provides a piece of the puzzle for 
understanding the architecture of control processes. Together, they indicate that control processes operate on abstract representations that include information about task-sets and the range of possible stimuli and responses.

\section{Sequential Modulations in the Temporal Flanker Task}

That sequential modulations of congruency effects were observed with the temporal flanker task at all indicates that they cannot be accounted for simply by enduring weightings on task relevant stimulus dimensions as proposed by the conflict monitoring hypothesis (Botvinick et al., 2001). Targets and flankers presumably used the same feature maps given that they differed only in terms of their relative timing, yet the influence of the flanker clearly depended on the congruency of the flanker on the previous trial. It is certainly possible that attention is set to the second item over the first, but this sort of tagging of items based on their relative order requires reference to the particular demands of the task, not just properties of the stimuli. In other words, sequential modulations appear to arise from the relative weighting of postperceptual representations, not stimulus features. This conclusion has obvious consequences for models of cognitive control. For example, the conflict monitoring model may have to be extended to include a mechanism for adjusting attention to post-perceptual dimensions in addition to stimulus dimensions.

Sequential modulations have been reported previously with priming tasks. However, in these cases (e.g., Kunde, 2003), the prime and the target stimulus differed according to their size, making it possible to suppress the prime based on stimulus properties. It should be noted that the congruency effects were somewhat larger in the current experiments. For example, in Kunde's (2003) Experiment 1, the magnitude of the congruency effect was $72 \mathrm{~ms}$, whereas in Experiment 1 of the present study, the magnitude was $152 \mathrm{~ms}$. Both effects are much larger than what is typically observed with standard flanker tasks, which is usually about 40 ms (e.g., Eriksen \& Eriksen, 1974), suggesting that the shared location of the target and flanker may make the flanker (or prime) harder to ignore. This may reflect the role of spatial attention in determining the magnitude of the congruency effect in the standard (Eriksen) flanker task, or it may be because the flankers (or primes) were much more confusable with the targets in tasks in which they appear at the same location. However, it is difficult for either spatial attention or confusability to account for sequential modulations in the current task-that is, why should having an incongruent stimulus on the previous trial help differentiate the target and flanker on the current trial? It is especially difficult to account for set-level sequential modulations with this sort of explanation.

\section{Set-Level Sequential Modulations}

By using four-choice tasks, we were able to differentiate between item-based changes in processing and set-based changes in processing. Our primary analyses of set-level processes indicated robust sequential modulations that did not stem from repetitions of any items from the previous trial. In this way, the findings rule out the possibility that sequential modulations simply reflect bindings between stimulus and response features, as in previous studies (e.g., Akçay \& Hazeltine, 2007, 2008, 2011; Funes et al., 2010; Notebaert \& Verguts, 2008; Ullsperger et al., 2005; Wendt et al., 2006). The conclusions of these previous studies are extended to a task in which the relevant and irrelevant information share the same features. One could argue that across all of the experiments, the source of the irrelevant information was always the same-the first item-yet both local and global control were observed, with the form of control depending on the construction of the task sets. Like the findings described in the previous section, this result argues against a model of control that only has at its disposal the weightings of various sources of inputs.

Previous work has indicated that individual features are not essential for other forms of sequential effects. In a study of action-effect binding, Dutzi and Hommel (2009) demonstrated biases to produce an alternating response to an alternating stimulus in two-choice tasks. Thus, the effects of action-effect bindings can be observed even when stimulus repetitions are eliminated from the analyses. It is unclear how such an account could be applied to comparisons like those reported in the present study in which the analyzed trials involve nonrepeating stimuli and responses. However, Dutzi and Hommel's finding that the performance of a trial involving one set of stimulus and response features can affect the performance of a subsequent trial involving nonrepeating stimulus and response features is consistent with the finding that sequential effects can extend beyond the item-level.

In the present study, the data argue against individual features playing a critical role in the implementation of cognitive controlthat is, control being applied to particular stimuli or features (see Blais et al., 2007) —at least the features in question can appear both as relevant and irrelevant sources of information. The effects of a particular flanker's history provided little evidence that control was implemented on specific items. When the flanker on the current trial had previously been an incongruent flanker, the magnitude of the congruency effect was consistently smaller than when flanker had previously been a target, which can be interpreted as reflecting item-based control. However, the pattern stemmed only from differences on congruent trials. Because targets and flankers were identical on congruent trials, the effect observed on these trials likely stems from negative priming of the target rather than changes in the influence of the flanker. On the incongruent trials, in which the flanker and target differed, the RTs were not affected by the history of the flanker. Thus, there is little evidence for item-based control.

Instead, sequential modulations appear to be specific to a particular task set, provided that the distinction between the sets if sufficiently salient, either because of their perceptual properties or because of the task demands. In this sense, the present findings are consistent with those of Notebaert and Verguts (2008), who examined sequential modulations within and between the Simon and SNARC tasks (see also, Kiesel et al., 2006). They found that sequential modulations crossed tasks when the two tasks used the same relevant information (i.e., both tasks required the participant to evaluate whether the target was italicized), but was task-specific when the two tasks involved different relevant information (i.e., responses in the Simon task were based on color; responses in the SNARC task were based on italicization). In short, sequential modulations were observed when participants were encouraged to conceptualize the two tasks as the same, because they were per- 
forming the same operation on the two sets of stimuli. When the two tasks required different judgments, local control was observed.

While previous research has argued that different sources of information are controlled separately (Akçay \& Hazeltine, 2011; Egner et al., 2007; Funes et al., 2010; Notebaert \& Verguts, 2008; see also, Schumacher et al., in press), the present study argues that the sources themselves do not define the domains of control. Based on our findings from Experiments 2, 3, and 4, we argue that whether the relevant stimulus feature changes is not the critical factor in determining whether control acts locally or globally. Rather it is the salience of the boundary between the two tasks that determines the boundaries of control processes. This may provide clues as to why Freitas et al. (2007) obtained evidence for global control despite using two tasks with distinct forms of conflict and distinct sets of stimuli. With the exception of their color Stroop task, they used four-choice stimulus sets that increased the heterogeneity within the tasks, and the task switched randomly, thereby possibly reducing the salience of the task boundaries.

In sum, the present findings indicate that, under the appropriate conditions, a stimulus' membership to a particular set determines the control parameters for that trial. This result provides further evidence that control processes are sensitive to task sets even under conditions that produce minimal task-switching costs. In other words, control appears to be implemented at the level of the task set, not the level of the stimulus features. Moreover, there is evidence that control parameters persist across switches, indicating that distinct parameters are maintained for the two tasks.

\section{Accounting for Set-Level Sequential Modulations}

The findings suggest that the control processes responsible for sequential modulations are able to act on representations that include abstract, or at least relative, information (e.g., increase the weighting of the second stimulus) and that they do so in a set-selective fashion. Thus, we propose that sequential modulations may reflect adjustments in task representations that include, in addition to the stimulusresponse mappings, weightings of the target and flanker. As participants perform the task, they develop a task representation that changes dynamically with each trial. After incongruent trials, the relative weighting of the flanker is decreased, and after congruent trials, the relative weighting of the flanker is increased. Critically, because these changes occur at the level of task representation, they can refer to items with respect to their role in the task rather than in terms of primitive perceptual properties. That is, adjustments can be made to the processing of the target or the flanker without specifying its particular stimulus properties.

Evidence for this sort of task representation has been proposed to account for other phenomena relating to stimulus-response translation. An obvious domain is the task-switching literature. Task switch costs are robust even when none of the stimulus features are repeated across the switch (e.g., Rogers \& Monsell, 1995), indicating that it is associated with changes in the task set rather than in the mappings of individual stimuli. Furthermore, Waszak, Hommel, and Allport (2003) demonstrated that these costs are moderated by the retrieval of a task representation, as opposed to a particular response association, that was previously associated with the current stimulus. The history of the individual stimuli does not appear to be critical in the present case, most likely because there were many fewer possible stimuli that re- peated much more often. Nonetheless, the notion of a retrieved task is highly similar to what we propose.

As pointed out by Spapé and Hommel (2008), these findings underscore a similarity between the control accounts and episodic accounts of sequential modulations. The two classes of explanation are similar if one assumes that episodic retrieval involves not just priming of particular responses but the reinstantiation of control parameters from previous trials. Spapé and Hommel (2008) used a Stroop-like conflict task in which an irrelevant auditory stimulus showed a sequential modulation based on the previous trial, but only when the voice (i.e., the speaker) of the irrelevant stimulus remained the same. They concluded that the modulations depend on the similarity of the contexts, which serve as cues that lead to the retrieval of the task representation. Again, this is highly consistent to our proposal, especially if one views the relationship between the target and flanker as a potential contextual cue. As we discuss below, the similarity of two contexts appears to depend on the range of possible events and their associated responses, not just the particular stimulus features present on the two trials. That is, contexts may be similar because of what they signify; two stimuli indicating responses with the same hand, for example, may be viewed as providing a similar context, whereas the same two stimuli, if they indicate responses with different hands, may be less likely to lead to the retrieval of the same task representation.

\section{Determining Set Boundaries}

In contrast to previous studies, Experiments 2 and 4 demonstrated global control when two sets of stimuli involved distinct relevant dimensions. Note that in the previous studies reporting local control (e.g., Akçay \& Hazeltine, 2008; Egner et al., 2007; Funes et al., 2010; Notebaert \& Verguts, 2008; Wendt et al., 2006), the distinction between the dimensions was task relevant: one dimension indicated the correct response and the other provided the conflict. Thus, to perform the task correctly, participants had to ignore one dimension and attend to the other. In contrast, in the present experiments, relevant and irrelevant information was presented along the same dimension. This may explain why global control could be obtained: there was no reason for participants to differentiate between the two sources of information, except when the distinction was salient (Experiment 1) or task-relevant (Experiment 3).

We do not claim to have mapped out the range of factors that can contribute to the determination of the task boundaries; this endeavor is beyond the scope of the present research. For example, it is possible that instructional manipulations can affect task boundaries, or that they are influenced by the environmental consequences of the responses. For the present, we conclude that the boundaries of control are not strictly related to the stimulus dimensions, but rather reflect the current task demands (i.e., whether a feature is relevant for determining the appropriate response or how salient one form of variation is compared to other sources of variability within the stimulus set).

The boundaries of sequential modulations have been used to draw inferences about the architecture of control processes (e.g., Blais et al., 2007; Egner, 2008; Funes et al., 2010; Notebaert \& Verguts, 2008). The present findings indicate that control processes operate on postperceptual representations and are not tied to stimulus properties. Thus, one might argue that rather than attempt to delineate the structure of control processes, a better starting point for understanding 
performance might be to determine the factors that lead to the representation of distinct task sets. Such an approach might attempt to find converging measures of task-set boundaries. For example, task set boundaries may affect the types of associations that can be formed between stimuli (see Keele, Ivry, Mayr, Hazeltine, \& Heuer, 2003) or may alter compatibility and repetition effects between consecutive responses (Schuch \& Koch, 2004).

Human behaviors are elaborate and complex. We frequently switch from one task to another to achieve subgoals and coordinate the demands of real-world situations, giving rise to the need for control processes to organize our actions and connect related responses. Critically, a particular task may involve diverse inputs that vary along multiple dimensions. That is, the task may cohere around a particular set of goals or concepts but vary in terms of the modalities of the stimuli and responses. Thus, effective control must not be driven by accidental properties of the incoming information; it should be based on the individual's goals. The present findings indicate that control processes abide by the organization of flexibly determined task-sets.

\section{References}

Akçay, Ç., \& Hazeltine, E. (2007). Feature-overlap and conflict monitoring: Two sources of sequential modulations. Psychonomic Bulletin \& Review, 14, 742-748.

Akçay, Ç., \& Hazeltine, E. (2008). Conflict adaptation depends on task structure. Journal of Experimental Psychology: Human Perception and Performance, 34, 958-973.

Akçay, Ç., \& Hazeltine, E. (2011). Domain-specific conflict adaptation without feature repetitions. Psychonomic Bulletin \& Review, 18, 505-511.

Blais, C., Robidoux, S., Risko, E. F., \& Besner, D. (2007). Item-specific adaptation and the conflict-monitoring hypothesis: A computational model. Psychological Review, 114, 1076-1086.

Botvinick, M. M., Braver, T. S., Barch, D. M., Carter, C. S., \& Cohen, J. D. (2001). Conflict monitoring and cognitive control. Psychological Review, 108, 624-652.

Dutzi, I. B., \& Hommel, B. (2009). The microgenesis of action-effect binding. Psychological Research, 73, 425-435.

Egner, T. (2008). Multiple conflict-driven control mechanisms in the human brain. Trends in Cognitive Science, 12, 374-380.

Egner, T., Delano, M., \& Hirsch, J. (2007). Separate conflict-specific cognitive control mechanisms in the human brain. NeuroImage, 35, 940-948.

Egner, T., \& Hirsch, J. (2005). Cognitive control mechanisms resolve conflict through cortical amplification of task-relevant information. $\mathrm{Na}$ ture Neuroscience, 8, 1784-1790.

Eriksen, B. A., \& Eriksen, C. W. (1974). Effects of noise letters upon the identification of a target letter in a nonsearch task. Perception and Psychophysics, 16, 143-149.

Fernandez-Duque, D., \& Knight, M. (2008). Cognitive control: Dynamic, sustained, and voluntary influences. Journal of Experimental Psychology: Human Perception and Performance, 34, 340-355.

Freitas, A. L., Bahar, M., Yang, S., \& Banai, R. (2007). Contextual adjustments in cognitive control across tasks. Psychological Science, 18, 1040-1043.

Funes, M. J., Lupiáñez, J., \& Humphreys, G. W. (2010). Analyzing the generality of conflict adaptation effects. Journal of Experimental Psychology: Human Perception and Performance, 36, 147-161.

Gratton, G., Coles, M. G. H., \& Donchin, O. (1992). Optimizing the use of information: Strategic control of activation and responses. Journal of Experimental Psychology: General, 121, 480-506.

Hommel, B. (1998). Event files: Evidence for automatic integration of stimulus-response episodes. Visual Cognition, 5, 183-216.
Hommel, B., Proctor, R. W., \& Vu, K. L. (2004). A feature-integration account of sequential effects in the Simon task. Psychological Research, 68, 1-17.

Jacoby, L. L., Lindsay, D. S., \& Hessels, S. (2003). Item-specific control of automatic processes: Stroop process dissociations. Psychonomic Bulletin \& Review, 10, 638-644.

Keele, S. W., Ivry, R. B., Mayr, U., Hazeltine, E., \& Heuer, H. (2003). The cognitive and neural architecture of sequence representation. Psychological Review, 110, 316-339.

Kiesel, A., Kunde, W., \& Hoffman, J. (2006). Evidence for task-specific resolution of response conflict. Psychonomic Bulletin \& Review, 13, 800-806.

Kornblum, S., Hasbroucq, T., \& Osman, A. (1990). Dimensional overlap: Cognitive basis for stimulus-response compatibility-A model and taxonomy. Psychological Review, 97, 253-270.

Kunde, W. (2003). Sequential modulations of stimulus-response correspondence effects depend on awareness of response conflict. Psychonomic Bulletin \& Review, 10, 198-205.

Kunde, W., \& Wühr, P. (2006). Sequential modulations of correspondence effects across spatial dimensions and tasks. Memory \& Cognition, 34, 356-367.

Logan, G. D. (2003). Executive control of thought and action: In search of the wild homunculus. Current Directions in Psychological Science, 12, 45-48.

Mayr, U., Awh, E., \& Laurey, P. (2003). Conflict adaptation effects in the absence of executive control. Nature Neuroscience, 6, 450-452.

Milliken, B., Joordens, S., Merikle, P. M., \& Seiffert, A. E. (1998). Selective Attention: A reevaluation of the implications of negative priming. Psychological Review, 105, 203-229.

Monsell, S., \& Driver, J. (2000). Banishing the control homunculus. In S. Monsell \& J. Driver (Eds.), Attention and Performance XVIII: Control of Cognitive Processes (pp. 3-32). Cambridge, MA: MIT Press.

Notebaert, W., \& Verguts, T. (2008). Cognitive control acts locally. Cognition, 106, 1071-1080.

Rogers, R., \& Monsell, S. (1995). The costs of a predictable switch between simple cognitive tasks. Journal of Experimental Psychology: Human Perception and Performance, 124, 207-231.

Schuch, S., \& Koch, I. (2004). The costs of changing the representation of action: Response repetition and response-response compatibility in dualtasks. Journal of Experimental Psychology: Human Perception and Performance, 30, 566-582.

Schumacher, E. H., Schwarb, H., Lightman, E., \& Hazeltine, E. (in press). Investigating the modality specificity of response selection using a temporal flanker task. Psychological Research.

Spapé, M. M., \& Hommel, B. (2008). He said, she said: Episodic retrieval induces conflict adaptation in an auditory Stroop task. Psychonomic Bulletin \& Review, 15, 1117-1121.

Stürmer, B., Leuthold, H., Soetens, E., Schröter, H., \& Sommer, W. (2002). Control over location-based response activation in the Simon task: Behavioral and electrophysiological evidence. Journal of Experimental Psychology: Human Perception and Performance, 28, 1345-1363.

Tipper, S. P., \& Cranston, M. (1985). Selective attention and priming: Inhibitory and facilitatory effects of ignored primes. Quarterly Journal of Experimental Psychology, 37, 591-611.

Ullsperger, M., Bylsma, L. M., \& Botvinick, M. M. (2005). The conflict adaptation effect: It's not just priming. Cognitive, Affective \& Behavioral Neuroscience, 5, 467-472.

Waszak, F., Hommel, B., \& Allport, A. (2003). Task-switching and longterm priming: Role of episodic stimulus-task bindings in task-shift costs. Cognitive Psychology, 46, 361-413.

Wendt, M., Kluwe, R. H., \& Peters, A. (2006). Sequential modulations of interference evoked by processing task-irrelevant stimulus features. Journal of Experimental Psychology: Human Perception and Performance, 32, 644-667.

Received October 7, 2010

Revision received February 28, 2011

Accepted May 23, 2011 